\title{
Studie: Das Handy als alltägliches Lifestyle-Objekt
}

\author{
Markus Czok, Marc Gurczik
}

\section{Einleitung}

Die rasante Entwicklung auf dem Handymarkt ist stets in den Medien, doch selten wird über die Nutzer berichtet, die das Handy mittlerweile als alläglichen Gebrauchsgegenstand akzeptiert haben. Diese Studie soll einen Überblick schaffen, über die Gewohnheiten von Handynutzern und die Veränderungen deren Kommunikations- und Konsumverhaltens, die aus der Nutzung eines Mobiltelefons resultieren. Zusätzlich werden auch Personen der Altersgruppe 50+ auf ihr Kommunikationsverhalten hin untersucht und nach ihren Bedürfnissen an ein Handy und dessen Dienste befragt. Dazu werden zwei Umfragen über den täglichen Gebrauch des Mobiltelefons durchgeführt. Dabei ist zu beachten, dass bei einer Online-Umfrage über das Internet keine repräsentativen Ergebnisse über die Gewohnheiten der Altersgruppe 50+ erzielt werden können. Für die Altersgruppe 50+ kann im Allgemeinen nicht zu den, mit dem Internet vertrauten Personengruppen gezählt werden. Somit ist es notwendig, die Umfrageergebnisse der Online-Umfrage mit einer, auf der Straße durchgeführten Befragung zu ergänzen, um zusätzliche Informationen von dieser Altersgruppe in Bezug auf deren Kommunikationsverhalten und deren Anforderungen an neue Produkte und Dienste zu erhalten. Die Online-Befragung richtet sich vorwiegend an die jüngere Generation im Alter von 12-25 Jahren und enthält zusätzliche Fragen über die Nutzung von Diensten und Funktionen, wie z.B. die Nutzung von MMS oder einer integrierten Digitalkamera. Die Auswertung der Umfragen wird dann nach Altersgruppen geordnet vorgenommen, wobei das Hauptaugenmerk auf der Altersgruppe von 12-25 Jahren und von 50 Jahren aufwärts liegt. Zusätzlich werden noch weitere Studien aus dem Internet einbezogen um ein allgemeingültigeres Ergebnis zu erzielen. Dabei wird darauf geachtet, dass nur seriöse Studien, wie die jährlich durchgeführte JIM-Studie vom Medienpädagogischen Forschungsverbund Südwest in Kooperation mit der Landesanstalt für Kommunikation Baden-Württemberg (LFK) und der Landeszentrale für private Rundfunkveranstalter Rheinland-Pfalz (LPR) verwendet werden.

\subsection{Applikationen - Services - Kosten}

Bei der Befragung werden hauptsächlich drei verschiedene Arten von Funktionen untersucht. Zum einen Funktionen, die unmittelbar mit der Kommunikation über das Mobiltelefon in Verbindung stehen. Neben dem klassischen Telefonieren sind das vor allem SMS, MMS und Internet. Obwohl man die beiden letzteren auch teilweise zu den Zusatzfunktionen eines Telefons zählen kann, die nur noch bedingt zur Kommunikation mit anderen Menschen dienen.

$\mathrm{Zu}$ diesen Zusatzfunktionen zählen vor allem die Kamera, der MP3-Player oder auch das Radio, sowie die
Möglichkeiten Klingeltöne, Logos und Spiele auf das Mobiltelefon laden zu können.

Als dritter wichtiger Punkt neben diesen Funktionalitäten spielt die Möglichkeit zur Individualisierung eine entscheidende Rolle. Die vorher genannte Klingeltöne und Hintergrundbilder sind ein Teil der Individualisierung. Eine weitere Möglichkeit ist das Austauschen der Oberschale.

Diese Befragung soll die grundsätzlichen Unterschiede in der Nutzung von Applikationen und Diensten zwischen den nachfolgend beschriebenen Zielgruppen zu Tage fördern. Der ursprüngliche Sinn des Mobiltelefons lag darin zu telefonieren, d.h. Sprachdienste standen im Vordergrund. Das ändert sich nun mit den nachfolgenden Generationen. Neue Dienste und Applikationen treten in den Vordergrund.

Beim Mobilfunk werden grundsätzlich zwei verschiedene Arten von Verträgen unterschieden. Die Verträge mit monatlicher Abrechnung (Postpaid) und die mit Vorauszahlung (Prepaid). Bei den Postpaid-Verträgen gibt es eine Reihe an Variationen und die Gebühren pro Gesprächseinheit können stark variieren. Je nach Vertragsart unterscheiden sich die Grundgebühren der Verträge, sowie die Minutenpreise. Einige Anbieter bieten Minutenpakete an, anderen zeit- und ortsabhängige Vergünstigungen. Die zusätzlichen Tarifoptionen, wie etwa die Gebührentaklung, sind zusätzliche Eckpunkte eines Vertrages. Anhand der Umfragen soll festgestellt werden, ob es für die verschiedenen Altersgruppen eine Tendenz zu einer spezifischen Vertragsart gibt.

Seit der Einführung flächendeckender digitaler Mobilfunknetze im Jahre 1990 haben sich im Laufe der Zeit neben der Telefonie, verschiedene Zusatzdienste entwickelt und etabliert. Während der Markt noch nicht bereit und auch Geräte und Ausrüstung anfangs noch nicht dazu geeignet waren, ergaben sich später durch immer fortschreitende Miniaturisierung von Bauteilen und Erhöhung von Prozessorleistung und Speicherkapazitäten ganz neue Möglichkeiten.

Einen Siegeszug hat dabei die SMS (Short Message Service) gemacht. Sie wurde anfänglich als Abfallprodukt kostenlos angeboten. Als die Netzbetreiber aber dann die wachsende Beliebtheit erkannten, wurde die SMS kostenpflichtig. Mittlerweile generiert der SMS-Dienst die höchsten Erträge der Netzbetreiber. Im Jahre 2003 wurde rund 16 Milliarden SMS in Europa verschickt. SMS liegen bei einem Preis zwischen 9 und 19 Eurocent. Verwandte Dienste sind EMS (Enhanced Message Service) und vor allem MMS (Multimedia Messaging Service). Die MMS bietet die Möglichkeit, neben Text auch Bilder, Ton und Videos zu versenden. Die Kosten zum Versenden einer MMS sind abhängig von der Größe der MMS und beginnen bei ca. 39 Eurocent.

Im Jahr 2002 begannen die Hersteller, Digitalkameras in ihre Mobiltelefone einzubauen. Mittlerweile ist kaum 
noch ein Telefon auf dem Markt zu finden, das keine Digitalkamera hat. Die Qualität die anfänglich sehr schlecht war, hat sich mittlerweile auch auf ein akzeptables Maß gesteigert. Einher, mit der Möglichkeit Fotos aufzunehmen, geht die Möglichkeit Bilder auf dem Telefon zu speichern, sowie Musik und Videos. Viele Mobiltelefone besitzen zudem auch einen integrierten Musikplayer oder ein Radio. Da der interne Speicher in Mobiltelefonen nicht sehr groß ist, kommt immer mehr der Trend auf, Speicherkarten in die Mobiltelefone einzusetzen. Damit ist eine enorme Erhöhung der Speicherkapazität verbunden. Diverse Anbieter bieten die Möglichkeit Klingeltöne und Logos (Hintergrundbilder) auf das Mobiltelefon zu laden. Hier gibt es verschiedene Kostenmodelle. Es gibt die Möglichkeit einzelne Bilder oder Musikstücke herunterzuladen oder ein so genanntes Monatsabo abzuschließen und monatlich eine gewisse Anzahl von Bildern oder Klingeltönen herunterzuladen. Die Kosten liegen dabei einmalig bei ca. 1 bis 3 Euro oder monatlich bei ca. 5 Euro.

Durch steigende Übertragungsgeschwindigkeiten im Mobilfunk, durch HSCSD, GPRS und UMTS werden nicht nur Webanwendungen und Internet interessanter, es können auch viele neue Dienste realisiert werden, wie etwa Videostreaming oder Videotelefonie. Neben dem Internet gibt es auch die Möglichkeit E-Mails und Faxe zu versenden. Die Preise für Datendienste liegen bei GPRS als volumenbasiertem Übertragungsverfahren zwischen 9 und 19 Eurocent pro 10kb-Datenpaket.

Die meisten Mobiltelefone sind heutzutage mit einem Java-Interpreter ausgestattet, der es ermöglicht Java-Anwendung auf dem Mobiltelefon laufen zu lassen. Der Funktionsumfang dieser Java-Laufzeitumgebung ist jedoch sehr eingeschränkt. Es gibt die Möglichkeit Spiele und Programme auf das Telefon zu laden. Einige davon gibt es als Freeware im Internet, andere müssen kostenpflichtig aufs Mobiltelefon geladen werden. Hier liegen die Kosten ungefähr so hoch wie bei den Klingeltönen und Logos.

Mit der fortschreitenden Miniaturisierung der Terminalkomponenten und Erweiterung der Möglichkeiten der Mobiltelefone werden immer weitere kostenpflichtige Zusatzdienste (Value-Added Services) auftauchen.

\subsection{Erläuterung der Zielgruppen}

Um eine möglichst breite Grundlage zur Auswertung und somit eine allgemeingültige Aussage zu erhalten, werden zwei Zielgruppen für die Umfrage definiert die relativ breit gefächert sind.

Die erste Zielgruppe sind Jugendliche, die ein Handy besitzen im Alter von 12-25 Jahren. Zur Befragung dieser Personengruppen wurde eine Online-Umfrage durchgeführt, da viele Jugendliche einen Zugang zu diesem Medium haben und somit auch die nötige Resonanz erreicht wird. Da die meisten Jugendlichen ein Handy besitzen und auch die Zielgruppe vieler Geschäftsideen, wie dem Versenden von Logos oder Klingeltönen sind, wurde in der Online-Umfrage zusätzlich auf Nutzung derartiger Zusatzfunktionalitäten eingegangen. Ergänzend dazu wurden noch Jugendliche am Berliner Alexanderplatz befragt, da sich an der Online-Umfrage kaum Jugendliche im Alter von 12-20 Jahren beteiligt haben. Dafür wurde der gleiche Fragebogen wie bei der Online-Umfrage verwendet.
Die zweite Zielgruppe beinhaltet Personen über 50 Jahren. Jedoch wurden in dieser auch Personen befragt die kein Handy besitzen, um die Gründe dafür zu erfahren. Für diese Generation wurde ein Fragebogen erstellt, der speziell auf diese Zielgruppe ausgerichtet ist. Diese Unterteilung wurde gewählt, um eine spätere Gegenüberstellung der beiden Altersgruppen zu ermöglichen. Somit können die verschiedenen Interessen der Personen aus den beiden Zielgruppen an dem Design, der Marke oder beispielsweise den Funktionen eines Handys in Erfahrung gebracht werden. Außerdem können so mögliche Interessenschwerpunkte der Zielgruppen ausgewertet und gegenübergestellt werden. Auf die daraus entstehenden Erfahrungswerte wird in den folgenden Kapiteln näher eingegangen.

\subsection{Die Umfrage}

Wie schon zuvor erwähnt, wurden zwei verschiedene Umfragen durchgeführt. Die Online-Umfrage für die Jugendlichen (siehe Anhang 1.1) wurde auf einer Webseite zugänglich gemacht. Zusätzlich wurden noch diverse EMails mit dem Link auf diese Webseite verschickt, damit genügend Teilnehmer bei dieser Umfrage mitmachen. Da sehr wenig Jugendliche im Alter von 12-20 an der OnlineUmfrage teilnahmen, wurde am Berliner Alexanderplatz das Ergebnis mit Hilfe einer Straßenumfrage ergänzt. In diesen Umfragen sollten die beliebtesten Handymarken, Vertragspartner und Zahlungsarten der Jugendlichen und jungen Erwachsenen ermittelt werden. Zusätzlich gab es aber noch eine Frage die Auskunft darüber geben sollte, wer die Handyrechnungen bezahlt.

Ein weiterer Themenkomplex in dieser Umfrage waren die Funktionen, wobei sowohl nach der Wichtigkeit als auch nach der Häufigkeit der Nutzung der verschiedenen Funktionen gefragt wurde. Dabei fand das Austauschen und Herunterladen von Klingeltönen, Musik oder Bildern besondere Beachtung. Aber auch die Nutzung der Möglichkeit zur persönlichen Anpassung des Handys in Bezug auf Hintergrundbilder, eigene Klingeltöne oder auswechselbare Oberschalen wurde untersucht.

Um die Auswirkungen eines Verlustes des Handys auf die sozialen Kontakte der betroffenen Person zu erfassen, wurde abschließend noch gefragt, ob eine Kopie des Adressspeichers existiert oder ob alle Telefonnummern dann auch verloren sind.

Weiterhin war es möglich noch Anregungen zu diesem Thema in ein Textfeld zu schreiben. Diese Umfrageergebnisse wurden zentral auf dem Server abgelegt und mussten dann nicht erst digitalisiert werden. Die Ergebnisse der Straßenumfrage wurden dann hinzugefügt.

Mit Hilfe der Online-Umfrage konnten auch, wie erwartet, nicht genügend Personen der zweiten Zielgruppe, die der über 50-jährigen, angesprochen und befragt werden. Deshalb wurde zusätzlich eine Umfrage für diesen Personenkreis auf dem Berliner Alexanderplatz durchgeführt. Für diese Umfrage wurden Fragebögen angefertigt, die speziell auf die Gewohnheiten dieser Personengruppe eingehen. Die Fragebögen wurden nochmals in zwei Gruppen unterteilt: Ein Fragebogen für Personen die ein Handy besitzen und ein Fragebogen für Personen die kein Handy besitzen. Dies ist notwendig um auch die Wünsche und Erwartungen an ein Handy von der Personengruppe 
zu erhalten, die (noch) kein Handy besitzt. Es wurde außerdem nach dem Grund gefragt, warum diese Personen sich noch kein Handy zugelegt haben oder warum sie sich kein Handy zulegen wollen. Die Umfrage erfasste ebenfalls das Alter und Geschlecht der Befragten, um eventuelle Tendenzen diesbezüglich herauszustellen. Auch hier wurden die beliebtesten Vertragspartner, Handyhersteller und Zahlungsarten, also Postpaid oder Prepaid, ermittelt. Weitere Fragen sollten Auskunft über die Gewohnheiten in Bezug auf die generelle Nutzung des Mobiltelefons der Befragten geben, also ob diese das Handy stets dabei und eingeschaltet haben und welche Funktionen des Handys am häufigsten genutzt werden. Die letzte Frage in dem Teilbereich für die Handybesitzer geht auf die Wünsche an das zukünftige Handy für die befragte Personengruppe ein. Also ob das Handy speziell für ältere Personen angepasst werden muss und wie diese Anpassungen aussehen könnten.

\section{Mobiltelefon als alltägliches Lifestyle-Objekt}

Das Mobiltelefon hat sich immer mehr in unserem Alltag etabliert und ist heutzutage für viele Personen das wichtigste was sie mit sich herumtragen. Der Verlust des Handys stellt dann meist den „Weltuntergang" für den Betroffenen dar. Wie sehr sind wir von diesem Gebrauchsgegenstand abhängig? Und wie sehr wirkt sich das Handy auf soziale Kontakte aus? Ist es überhaupt noch vorstellbar ohne ein Handy zu leben?

Die Beantwortung dieser und weiterer Fragen hat sich diese Auswertung der Umfrage als Ziel gesetzt.

\subsection{Die Wahl der Marke}

Diese Studie untersucht unter anderem die Markentreue von Mobilfunknutzern, zum einen die Markentreue zum Gerätehersteller, andererseits die Markentreue zu einem bestimmten Netzanbieter oder Service-Provider. Es soll gezeigt werden, ob es eine altersabhängige Markentreue zu einem bestimmten Hersteller oder Netzbetreiber gibt. Die Umfrage liefert keine konkreten Ergebnisse dazu, da die Frage „Sind sie einer bestimmten Marke treu?“ zu sehr subjektiven Ergebnissen geführt hätte. Es soll daher durch andere Befragungsinhalte geklärt werden, ob eine Markentreue abgeleitet werden kann.

Während die Hersteller klar voneinander abzugrenzende Unternehmen sind, die mit ihren Produkten auf dem Markt gegeneinander konkurrieren, ist die Situation bei den Netzbetreibern und Mobilfunkprovidern nicht ganz so einfach. Die Netzbetreiber sind hier noch eindeutig zu unterscheiden. Sie bieten Dienste auf ihrem eigenen Mobilfunknetz an. Dagegen sieht die Situation bei den Serviceprovidern schon anders aus. Sie bieten Tarife unterschiedlich Netzbetreiber an, die sich erheblich von denen der Netzbetreiber unterscheiden können.

Auf dem Markt sind verschiedene Tarife und Tariffierungsmöglichkeiten anzutreffen. Grundsätzlich kann zwischen den Laufzeitverträgen und den Prepaid-Karten unterschieden werden. Laufzeitverträge sind meist mit einer Laufzeit von 24 Monaten verbunden, bieten dafür aber geringere Gebühren und ein subventioniertes Mobiltelefon. Bei der Prepaid-Karte zahlt man für das Mobiltelefon wesentlich höhere Preise und auch die Gebühren sind teurer, dafür ist man an keine Vertragslaufzeit gebunden.

Da die Umfrage nicht explizit nach dem ServiceProvider gefragt hat, kann an dieser Stelle nicht ermittelt werden, ob es sich um direkte Verträge bei den Netzbetreibern oder Verträge über Service-Provider handelt. Die Studie stellt die Marke des Netzes in den Vordergrund. Der Provider soll an dieser Stelle vernachlässigt werden.

Neben der Frage der Markentreue, ist ebenfalls die Frage von Interesse, ob es einen bevorzugten Mobiltelefonhersteller gibt oder ob es bei den verschiedenen Altersgruppen Auffälligkeiten bezüglich der Wahl des Handyherstellers gibt. Die Auswertungen der Umfrage, sowie die Erfahrungen bei der Umfrage können hierbei Aufschluss über verschiedene Fragestellungen geben.

Folgende Fragen versucht diese Umfrage zu beantworten:

- Gibt es Besonderheiten oder Auffälligkeiten bei einer der beiden Zielgruppen?

- Gibt es einen bevorzugten Hersteller innerhalb der beiden Zielgruppen?

- Gibt es einen bevorzugten Netzbetreiber innerhalb einer der beiden Zielgruppen?

- Ist eine Markentreue bei einer der beiden Zielgruppen erkennbar?

- Gibt es auffällige Unterschiede oder Gemeinsamkeiten zwischen beiden Zielgruppen bezüglich der vorher gestellten Fragen?

Die Umfrage bei den Jugendlichen hat eindeutig Siemens als beliebtesten Handyhersteller ergeben. Von den befragten Personen haben 19 Siemens als den bevorzugten Hersteller angegeben. Mit insgesamt 12 Stimmen nimmt Nokia hier den zweiten Rang in der Beliebtheit ein, dicht gefolgt von Sony-Ericsson mit 8 Stimmen. Weit abgeschlagen in dieser Umfrage sind die Hersteller Motorola mit 4 Stimmen und Sagem mit einer Stimme zu finden. Dieses Ergebnis war schon in etwa so zu erwarten, doch dass eine so deutliche Mehrheit Siemens und nicht Nokia bevorzugt, ist etwas überraschend. Auf die Frage, ob die Marke entscheidend für den Kauf eines Handys ist, haben nur wenige Befragte mit Ja geantwortet und einen Hersteller nennen wollen. Dies zeigt, dass sich Jugendliche nach den neuesten Entwicklungen am Markt richten und sich nicht auf einen Hersteller fixieren.

In der Zielgruppe der Jugendlichen hat nur ein kleiner Teil der Befragten die Nutzung von Funktionen wie dem Herunterladen von Klingeltönen oder Bildern in der Umfrage angegeben. Diese Gruppe lässt sich allerdings in Bezug auf das Alter eingrenzen, denn nur Jugendliche im Alter von 12 bis 14 Jahren nutzen diese Handyfunktionen. Mit zunehmendem Alter verliert sich jedoch das Interesse daran. Unter den Jugendlichen ab 15 Jahren aufwärts war nicht ein Befragter zu finden der für derartige Downloads Geld ausgibt. Durch den Wunsch nach Nutzung dieser Funktionen entsteht auch ein Wunsch nach einem aktuellen Gerät mit der richtigen Auflösung, um beispielsweise die heruntergeladenen Bilder in hoher Qualität darstellen zu können. Dies ergab auch das Ergebnis der Umfrage, in der fast alle Jugendlichen im Alter von 12 bis 14 ein 
aktuelles Handy von einem speziellen Hersteller als wichtig empfanden.

Eine weitere Auffälligkeit hat sich bei der Frage nach der Häufigkeit der Nutzung verschiedener Dienste/ Funktionen des Handys ergeben. Bei der Personengruppe ab 18 Jahren übertrifft die Nutzung des Telefondienstes die der Nutzung der SMS-Funktion erwartungsgemäß, wohingegen die Befragten von 13 bis 17 Jahren eindeutig das Versenden von Kurzmitteilungen vor dem Telefonieren favorisierten.

Die Teilnehmer, die eine Nutzung von Funktionen wie dem Fotografieren oder dem Hören von Musik über das Handy, angekreuzt hatten, gaben meist auch an, dass diese nur selten genutzt werden. Daraus lässt sich schließen, dass die zuvor erwähnten Funktionen eher als unwichtig unter den Jugendlichen angesehen werden.

Der beliebteste Provider unter den Befragten ist Vodafone mit 17 Stimmen. Doch hier ist der Abstand zum Zweitplatzierten o2 mit 10 Stimmen im Verhältnis größer als bei der Frage bezüglich des Handyherstellers weiter oben. T-Mobile landet mit 8 Stimmen auf dem dritten Platz. Es gab 6 Befragte die als Vertragspartner E-Plus und 3 die Debitel angaben. Es gab es nur einen Befragten der einen anderen Provider als die Üblichen, nämlich Hutchison, angegeben hat. Bei der Frage nach dem beliebtesten Hersteller sowie Provider haben sich bei den Teilnehmern der Umfrage keine geschlechtspezifischen Vorlieben ergeben. Es scheint also nicht geschlechterabhängig zu sein welche Marke und welcher Provider genutzt wird. Anders verhält es sich bei dem Altersabhängigkeiten. Zwar ist bei den Altersgruppen keine Tendenz bezüglich der Handymarke zu erkennen jedoch ist deutlich zu sehen, dass bei den jüngeren Befragten Prepaid sehr verbreitet ist. Bei den Personen unter 20 nutzt jeder zweite diese Art des Vertrages. Dies nimmt dann mit zunehmendem Alter ab, so dass, von den Personen über 24 Jahren, nur noch ein einziger mit einer Prepaid Karte telefoniert.

Bei den Befragten der zweiten Zielgruppe also Personen über 50 Jahren zeigt sich, dass auch innerhalb dieser Altersgruppe ein Trend zu Nokia und Siemens Handys besteht. Unter den Befragten gibt es 15 Nokia und 14 Siemens Nutzer. Die anderen Handyhersteller sind bei den Umfrageergebnissen weit abgeschlagen und werden nur von sehr wenigen Personen der befragten Altersgruppe genutzt. Es gab jeweils nur einen Befragten der ein Motorola, Sagem, Ericsson oder Alcatel Handy verwendet. Bei der Beliebtheit der Vertragspartner in dieser Altersgruppe befinden sich Vodafone und T-Mobile gleichauf an der Spitze mit jeweils 10 Stimmen. Weit dahinter rangieren andere Vertragspartner wie o2, Debitel und E-Plus mit jeweils nur 2 Stimmen. Da aber viele Befragte den Vertragspartner nicht wussten, ist dieser Teil nicht sehr aussagekräftig. Allerdings wussten die meisten Personen, ob sie mit einem Vertrag oder einer Prepaid Karte telefonieren. Die Auswertung der Umfrage für die Senioren ergab, dass wie bei den ganz jungen Befragten zwischen 12 und 19 die Nutzung einer Prepaid Karte sehr beliebt bzw. verbreitet ist. Die Teilnehmer begründeten diese Vorliebe häufig mit den niedrigeren Kosten. Auch dies lässt auf eine seltenere Nutzung des Mobiltelefons unter diesen Teilnehmern schließen. Bei den älteren Personen ist aber nicht wie bei den jüngeren eine Kostenkontrolle der Grund für die seltene Nutzung, sondern meist weil sie kein Bedarf haben. Auch hier ist eine Tendenz in Bezug auf das Alter der Befragten zu erkennen. Unter den Befragten zwischen 50 und 60 Jahren sind gerade einmal drei Personen mit einer Prepaid Karte zu verzeichnen. Dagegen telefonieren etwa 60 Prozent aus der Gruppe der Befragten im Alter über 60 Jahre mit einer Prepaid Karte. Bei näherer Nachfrage ergab sich als Grund dafür die geringeren Kosten. Diese Tendenz verdeutlicht, dass es für Ältere billiger ist eine wiederaufladbare Karte zu nutzen, wenn sie wenig telefonieren, nur angerufen werden oder das Handy nur für den Notfall brauchen.

Auffällig ist, dass viele der Befragten aus der älteren Zielgruppe den eigenen Provider nicht kennen und teilweise sogar nicht einmal den Hersteller des Handys. Aus diesen Tatsachen kann geschlossen werden, dass sich ältere Personen nicht viel mit ihrem Handy auseinandersetzen. Diese Erkenntnis wird durch die Ergebnisse bei der Frage nach genutzten Kommunikationsdiensten bestätigt. Bei dieser Frage hat ein Großteil der Teilnehmer ausschließlich die Nutzung des Telefondienstes angegeben. Nur wenige nutzen die SMS Funktion und wenn dann auch nur selten. Andere Funktionen werden nur von einem Befragten genutzt. Dieser ist allerdings als Ausnahme zu werten, denn diese Person hat das Handy sogar als Modem verwendet.

Die Frage ob die jeweilige Person das Handy oft dabei und auch eingeschaltet hat ergab, dass viele Personen dieser Zielgruppe das Handy oft zu Hause lassen und nur in ganz bestimmten Situationen mitnehmen. Auch diese Ergebnisse tragen zu dem Eindruck bei, dass ältere Leute das Handy nicht oft benutzen. Zusätzlich wird bei dieser Frage eine Tendenz in Bezug auf das Alter der Teilnehmer erkennbar. Je älter die Personen werden um so mehr von ihnen lassen das Handy oft zu Hause oder schalten es nicht ein.

Die Gruppe unter den Befragten die kein Handy besitzen, haben laut den Ergebnissen der Umfrage auch kein Interesse an einem Handy. Teilweise gab es regelrechte Abneigung gegen Handys von dieser Personengruppe. Auch das Argument der zu hohen Kosten ist zweimal gefallen. Überraschendes Ergebnis bei der Frage, ob sich die Personen ein Handy zulegen würden, wenn die Bedienung einfacher und übersichtlicher wäre, ist, dass etwa die Hälfte der Befragten sich für ein Handy entscheiden würden. Hauptargumente gegen den Kauf eines Handys sind die zu kleinen Tasten und die kleine Schrift auf einem winzigen Display. Aber auch die Menüführung wurde stark kritisiert in der Form, dass es zu viele unnötige Untermenüs gibt. Diese Tatsache und die Erfahrung, dass Dienste außer Telefonie und SMS selten genutzt werden, zeigt die Bereitschaft der älteren Personengruppe auf viele Zusatzfunktionen zu Gunsten der Übersichtlichkeit der Menüführung zu verzichten. Auch die Ergebnisse auf die Frage nach der Wichtigkeit der Marke des Handys tragen zu dem Eindruck der Unzufriedenheit der älteren Generationen mit der Menüführung bei. Denn die Entscheidung für eine spezielle Marke hängt bei dieser Personengruppe größtenteils von der Menüführung ab. Aus diesem Grund sind die meisten Älteren ihrer jeweiligen Handymarke treu, denn sie sind bereits mit dem Menü vertraut. 


\subsection{Design vs. Funktionalität}

Die Studie soll zeigen, dass gerade bei Jugendlichen das Design im Vordergrund steht, bei der Zielgruppe der älteren Menschen aber eher die Funktionalität. Jedoch beschreibt Funktionalität in diesem Fall nicht alle möglichen Funktionen die ein modernes Mobiltelefon zu bieten hat. Vielmehr sind hier Grundfunktionalitäten gemeint. Dazu gehört das Telefonieren und SMS schreiben. Es geht ebenfalls um die Bedienbarkeit der Telefone und ihrer einzelnen Funktionen. Design ist etwas weiter zu fassen, als die bloße äußere Erscheinung. Hier geht es ebenfalls um Funktionen, allerdings eher um neuartige Zusatzfunktionen, wie Kamera, MP3-Player usw. Design umfasst auch, die Möglichkeiten zur Individualisierung des Handys. Klingeltöne und Logos sind hier wichtige Indikatoren für eine Tendenz zum Design.

Bei der Zielgruppe der über 50-jährigen konnten über 17 Prozent derer, die ein Mobiltelefon besitzen, nicht sagen um welche Marke es sich handelt. Den Produkttyp konnte kein Einziger sagen. Jedoch gab es die beinahe einhellige Meinung, dass die Menüführung zu kompliziert sei, zu viele Funktionen am Handy seien, die Schrift auf dem Display zu klein sei und/oder die Tasten zu klein seien. Mitsamt dem Fakt, dass Telefonieren und in gewissem Maße SMS schreiben, fast die einzigen Funktionen sind, die Befragte dieser Altersgruppe nutzen, lässt den Schluss zu, dass Funktionalität hier an erster Stelle steht. Die Menschen möchten ein Mobiltelefon, welches die beiden Grundfunktionalitäten einfach und übersichtlich zur Verfügung stellt und fühlen sich mit den momentan am Markt befindlichen Geräten, nicht verstanden. Design spielte eine sehr geringe Rolle. Nur wenige Befragte erwähnten, dass ihnen die Größe des Handys wichtig sei.

Bei den jugendlichen Befragten, sah die Situation ganz anders. Hier konnten fast alle Befragten den Typen ihres Handys bestimmen. Es handelte sich dabei auch fast ausschließlich um aktuellere Modelle. Bei der Frage welche Funktionen die Jugendlichen nutzen, gaben 100 Prozent an das sie das Telefon und SMS benutzen. 35 Prozent der Befragten nutzen auch MMS, jedoch sehr selten oder ab und zu. 45 Prozent der Befragten nutzen die Handykamera. 11 Prozent nutzen das Mobiltelefon als MP3-Player. Immerhin 17 Prozent surfen im Internet, aber das auch sehr selten.

Die Frage nach der Individualisierung des Mobiltelefons ist bei den jugendlichen Befragten sehr unterschiedlich ausgefallen. 35 Prozent der Befragten sagten das sie ihr Mobiltelefon persönlich anpassen würden und das vor allem durch Klingeltönen (23 Prozent) und Logos (15 Prozent). Dabei waren vor allem die unter 18-Jährigen an diesen Möglichkeiten interessiert.

Die Möglichkeit mit dem Telefon Dateien auszutauschen nutzen ca. 15 Prozent und dann vor allem für Bilder (11 Prozent) und Musik (9 Prozent).

Letztendlich zeigt das, dass die Jugendlichen die Möglichkeiten, die ihr Mobiltelefon Ihnen bietet, vielmehr nutzen als die Menschen der älteren Generation. Diese brauchen vor allem das Telefonieren und das SMS schreiben. Es kann also gesagt werden, dass junge Menschen das Telefon eher als Designobjekt sehen, während ältere Menschen eher den ursprünglichen Nutzen des Telefons in den Vordergrund stellen.

\subsection{Der Kostenfaktor}

Während bei der Generation der über 50-jährigen lediglich 80 Prozent ein Mobiltelefon besaßen, haben nur 10 Prozent gesagt, das sie zusätzliche Dienste nutzen. Die zusätzlichen Dienste beschränkten sich zumeist auf die Weckfunktion des Mobiltelefons. Nur ein einziger Befragter hat das Telefon noch zusätzlich als Modem verwendet. 90 Prozent der Befragten dieser Zielgruppe nutzen das Mobiltelefon nur selten oder ab und zu zum Telefonieren. Lediglich 10 Prozent nutzen es oft oder sehr oft zum Telefonieren. 35 Prozent derer die ein Mobiltelefon besitzen nutzen es auch zum Schreiben von SMS-Nachrichten.

In dieser Altersgruppe war kein Befragter zu finden der für kostenpflichtige und teure Dienste Geld ausgegeben hätte. Ganz anders ist die Situation bei den Jugendlichen zwischen 12 und 25 Jahren. Bei der Umfrage ist ersichtlich geworden, dass nur Jugendliche unter 15 Jahren, das kostenpflichtige Herunterladen von Bildern oder Klingeltönen in Anspruch nehmen. Über dieser Altersgruppe ist kein Befragter zu finden, der diese Dienste in Anspruch nimmt. Während bei der Personengruppe 12-17 eher das Versenden von Kurzmitteilungen favorisiert wird, ist es bei den ab 18-jährigen eher das Telefonieren, obwohl immer noch sehr viele SMS versendet werden.

Die Kosten, die durch die Mobiltelefonnutzung entstehen, werden bei den über 18 Jährigen zu 79 Prozent von den Befragten selbst getragen. Ca. 15 Prozent sagt, dass die Eltern die Rechnung bezahlen. Bei 5 Prozent in dieser Altersgruppe teilen sich Befragte und deren Eltern die Telefonrechnung. Anders ist die Situation bei den unter 18 Jährigen. Hier geben insgesamt 80 Prozent an, dass sie sich die Telefonrechnung mit Ihren Eltern teilen. Bei 13 Prozent zahlen die Eltern die gesamte Rechnung und bei 5 Prozent zahlen die Befragten selbst.

Während bei den Jugendlichen ab 18 Jahren die Laufzeitverträge mit ca. 79 Prozent anzutreffen sind und Prepaid-Karten mit 21 Prozent, ist es bei den unter 18 Jährigen noch häufiger eine Prepaid-Karte. Da PrepaidKarten wesentlich höhere Tarife haben, schließen viele Eltern für ihre Kinder einen Vertrag ab, um so günstigere Tarife nutzen zu können.

\section{Fazit}

Die Auswertung der Umfrage bei den Jugendlichen hat ergeben, dass das Handy für die jüngeren Befragten dieser Zielgruppe eine größere Rolle spielt als bei den Jugendlichen ab 20 Jahren. Von den jüngeren nutzen mehr die Zusatzfunktionen, wie etwa das Herunterladen von Bildern oder Klingeltönen. Unter diesen Personen nimmt das Handy auch einen höheren Stellenwert ein, denn im Durchschnitt wird das Mobiltelefon von jüngeren häufiger genutzt als von Jugendlichen ab 20 Jahren. Vergleicht man die Nutzung zusätzlich mit der Zweiten Zielgruppe, den über 50-jährigen, fällt auch hier auf, dass jüngere Leute das Handy häufiger gebrauchen. Die Wichtigkeit eines aktuellen Handys wurde von den Jüngsten der Befragten als sehr hoch angegeben. Dies verdeutlicht den hohen Stellenwert des Mobiltelefons unter dieser Personengruppe und zeigt, dass es einen festen Platz in dem Leben eines 
Heranwachsenden einnimmt. Bei der Frage ob ein Verlust des Handy auch den Verlust aller Telefonnummern und Adressen zur Folge hat ergab sich, dass nicht einmal 70 Prozent der Jugendlichen eine Kopie des Telefonspeichers besitzen. Dies bestätigt die Behauptung, dass das Handy eine enorm wichtige Rolle im Leben von diesen Befragten einnimmt und zeigt das der Verlust Folgen für deren soziale Kontakte hat.

In dem Marktsegment des Handys für Personen $\mathrm{ab}$ 50 Jahren besteht jedoch nach der Umfrage noch starker Handlungsbedarf. Die Handyhersteller haben die Bedürfnisse der Personengruppe über 60 Jahren lange außer Acht gelassen und keine speziell angepassten Produkte für diese Zielgruppe entwickelt und auf den Markt gebracht. Diese Studie zeigt aber, dass es einen Bedarf nach einem einfachen auf wenige Funktionen reduzierten Handy gibt. Mit einem solchen Produkt können, wenn man nach den Ergebnissen der Umfrage geht, noch weitere Personen vom Nutzen eines Handys überzeugt werden. In dieser Altersgruppe ist allerdings ein starker Trend zu sehr seltener Nutzung des Telefons zu erkennen. Daraus lässt sich auf die geringe Wichtigkeit eines Mobiltelefons für die Befragten über 60 Jahren schließen. Diese Personen haben das Handy also noch nicht vollständig als alltäglichen Gebrauchsgegenstand akzeptiert. Diese Aussage wird auch von den Ergebnissen der Umfrage gestützt, die ergab, dass das Handy in dieser Altersgruppe hauptsächlich für den Notfall angeschafft wurde. Jedoch ist in diese Gruppe der Anteil der Personen, die das Handy meist zu Hause lassen sehr hoch. Dies stellt einen Widerspruch dar, denn wenn das Handy zu Hause liegt kann es im Notfall nicht helfen.

Die Studie zeigt, dass die Generationen, die von klein auf mit einem Mobiltelefon aufwachsen, mehr Interesse an der Nutzung des Handys zum Telefonieren oder an der Nutzung der Zusatzfunktionen besitzen. Von diesen Personen wird das Handy als Lifestyle-Objekt anerkannt.

Die Umfrage hat gezeigt, dass ältere Menschen eher auf die grundlegenden Funktionen Wert legen, als auf viele Spielereien. Es ist einerseits kein Bedarf an den angeboten Diensten dar oder die Dienste können aufgrund der immer komplexer werdenden Menüstruktur und den immer dichter werdenden Informationsangeboten nicht mehr erschlossen werden. Ältere Menschen benötigen ein Mobiltelefon bei dem sie die Grundfunktionen einfach und schnell bedienen können. Ganz im Gegensatz dazu stehen die jugendlichen Befragten, die neben den Grundfunktionen eines Mobiltelefons, nämlich Telefon und SMS, auch viele Zusatzfunktionen nutzen. Da der Mobilfunkmarkt in Deutschland nahezu gesättigt ist, versuchen die Anbieter nun mit kostenpflichtigen Zusatzdiensten auf den Markt zu kommen. Deshalb wird sich in Zukunft das Angebot an kostenpflichtigen Zusatzdiensten eher noch verstärken und gerade die heranwachsende Generation wird auf dieses Informationsangebot und diese Services anspringen.

Ein wichtiges Thema dürfte dabei auch die Finanzierung der Nutzung solcher Dienste stehen. Der Kostenfaktor von solchen Zusatzdiensten ist enorm. Viele Menschen unterschätzen diese Kosten und verschulden sich hoch. Die Umfrage hat gezeigt, dass gerade beim Nachwuchs die
Nutzung solcher kostenpflichtigen Zusatzdienste zunimmt. Das Handy wird immer mehr zum Lifestyle-Objekt. Aber Lifestyle-Objekte haben ihren Preis und so muss man auch beim Handy immer den Kosten-Nutzen Faktor im Auge behalten.

\section{Danksagung}

Wir danken Prof. Dr. Bernd Eylert für die Unterstützung bei der Ausarbeitung dieser Studie, sowie für die interessante Aufgabenstellung. Die Vorlesung Telekommunikation und Gesellschaft hat uns eine neue, interessante Perspektive auf die Telekommunikation gegeben, wo nur allzu oft nur auf technische Aspekte geachtet wird und die Frage nach den Wechselwirkungen mit der Gesellschaft zu kurz kommt.

\section{Autoren}

\author{
Markus Czok \\ Sittestraße 3c, 13437 Berlin \\ Tel. +493041718845 \\ mczok@gmx.de \\ Marc Gurczik \\ Am Comeniusplatz 5, 10243 Berlin \\ Tel. +491741800999 \\ marc.gurczik@web.de
}

